\title{
Geological Cloud Platform Based on Micro Service Architecture
}

\author{
Shi-Jie Peng, Zhi-Ting Zhang, Chong-Long Wu \\ CUG, Computer Science Department \\ Wuhan, China \\ Email: 616958232@qq.com
}

\begin{abstract}
This paper analyses the main problems of the data sharing and application services of geological survey in China, and analyses the current situation of the research of the domestic and foreign qualitative cloud and big data. China Geological Survey service targets and construction of mainstream cloud computing technology based on the geological survey data storage mechanism, logical architecture and application architecture, DOCKER is proposed based on geological micro heterogeneous system architecture for cloud service access, the era of big data processing, geological data processing, interpretation and application.
\end{abstract}

Keyword-Microservice; RESTFul; SaaS; Docker; geological data management.

\section{INTRODUCTION}

With the continuous deepening of the computer information technology and the geological industry, all aspects of geological work has been highly integrated with information technology. From data collection to data collation, indoor data management, data processing and interpretation, the phenomenon of map making, results analysis, resource prediction, investigation and evaluation, process simulation, and then to the achievements management, preservation, use and publication, even geological work management and decision-making, not closely linked with information technology.

Geological data as the carrier of geological information has the characteristics of multi source, multi type, multi dimension, multi dimension, multi temporal and multi subject [1]. The main source of geological data of outcrop observation, core description, logging and sampling tests, physical and mechanical testing, daily production records, hydrogeology survey, geophysical exploration, remote sensing, comprehensive research and edge geochemical exploration, and has all kinds of spit, investigation and research. Such a rich source of data, is bound to bring a wide range of data types and massive data. The multi temporal features of geological data are embodied in (1) the result of a certain stage of geological history. (2) geological survey work is according to the time of progressive stage. The main characteristics of multiple geological data is the value of its wide use, analysis of geological body, geological phenomena and geological analysis, and analysis of ore deposit characteristics, ore controlling conditions and metallogenic process, reserves calculation and comprehensive geological evaluation and geological mapping. Each subject is involved in a large number of different types of data, and the data processing process is very complex, in addition, a huge amount of property data, but also an important feature of geological data.

How to get good information from the perspective of geological information, good management and good use of so many sources, multi class, multi quantity, Dovi, multi situation and multi thematic geological data has become a common problem faced by geological information workers. At the same time, geological information workers and organizations at home and abroad have made a lot of work to try to solve the geological data management and application problems. The idea of industrial cluster based on geological data information service. To promote the management of geological data and service work (Wang Qianju et al..2011) based on the geological data information service cluster. In order to meet the demand of the network services of massive geological data, the proposed system (2014) is based on the service oriented architecture and virtualization technology. Geological cloud computing platform is designed. The principle of publishing, gathering and visiting of geological data service resources are analyzed. A geological cloud computing platform and a prototype system which can deploy data services, map services and computing services are implemented. Holdaway, Keith (2014) proposed a data driven model based on soft computing from foundation, data management, seismic attribute analysis, reservoir characteristics and simulation, drilling and completion optimization, reservoir management, production forecasting and optimization, exploration and Prospect of data analysis, big data (structured and unstructured) applications in the field of oil and gas exploration and development. The prospects and benefits of big data in the field of oil and gas exploration and development are expounded. Miao, Qu Honggang, Xu Zhe, Jinli (2014) [5] relying on the Ministry of land and resources of Geological Information Technology Key Laboratory of geo data technology research of GeoBDA experimental platform, through the processing technology stack, according to different types of data, the use of different components, realize the multiple geological data. GeoDB A including big data research based on Oracle architecture platform and Hadoop architecture based big data research experimental platform to solve the problem of data storage and management of geological data.

All of the above systems and researches have been solved in a certain extent and extent based on the network geological data sharing, application, and it is of great significance for the study of mineral exploration and development, geological bodies, geological phenomena and geological processes. However, these studies mainly focus on geological data sharing service platform construction, but now the geological information in the field or mineral industry status quo in addition to the lack of unified data service platform, between the various units and departments of heterogeneous systems and software flooding is difficult to form effective unity in a short period of time, their business has depth bound and 
system software their use. In this case, the establishment of a unified service platform for the data and the application of a step in place is difficult to implement.

According to the above mineral information problem, we propose a micro service system of geological cloud service system is built based on the geological data (raw data, basic data and data) and at the same time, computing integrated platform, the platform supports the existing local quality units and institutions application software and system in building a unified cloud, integrated service platform is compatible with existing and compatibility issues in software and systems.

\section{CLOUDWARE-NEW SOFTWARE FORM IN CLOUD ENVIRONMENT}

With cloud computing technology continues to mature, EC Amanon 2 App Engine Google and other representatives of the cloud services platform to flourish, more and more companies and individuals to reduce costs through the use of cloud computing services. There is a large part of cloud computing services for developers, and cloud computing services in SaaS and PaaS, SaaS layer users can only through the use of running on the cloud computing platform software indirectly enjoy cloud services. With the introduction of micro software services, cloud concept, cloud environment software is no longer a simple code entity, but a series of micro Hill services constitute the complex service, delivered through the Internet, reflect a more fit cloud environment software form, namely a cloud.

\section{A. Development Model of Cloudware}

In the process of traditional software development, developers need to build their own software development environment, such as IDE and compile tool chain, etc.. With the development of the Git and task management system, the development of cloud piece should reflect the development process for the cloud, cloud DE and compile the micro services completed the whole software development task, and task tracking using cloud collaboration software, cloud of software development from code and software engineering. At the same time, the development of the micro cloud should follow the concept of service, the software as much as possible into different components are encapsulated as services, reuse through the corresponding API interface, realize the evil solution of software module, and convenient continuous integration testing process. Especially in the team development software, similar to the Docker container technology can provide a reusable operating environment, flexible resource allocation and convenient integration test method. In the process of developing a cloud, the function call is not like traditional software such as operating system of library calls, but the micro service calls, the development of cloud oriented micro parts shall be in the form of service component, and does not depend on the specific operating system and hardware resources.

\section{B. Cloudware Deployment}

Cloud component deployment is actually micro service deployment. Currently Docker as the representative of the micro service container technology development is becoming more and more mature. Docke provides a series of container deployment tools for developers to provide a novel and convenient software integration test and deployment capabilities.

\section{CONSTRUCTION OF GEOLOGICAL CLOUD PLATFORM BASED ON DOCKER AND MICRO SERVICE}

\section{A. system Architecture}

In order to achieve the platform data and calculation of the distribution of inheritance, the system operation and maintenance of the unified management of the platform and the focus of functional services provided. Geological cloud micro service platform is logically divided into three service poly cocooning frame respectively geological data services, geological services and functional services. Each service is used by the micro poly service brought together, each service communicate through message queue in data service platform, a service cluster and poly poly support dynamic lateral extension, each service monomer stop influencing the work of service cluster and cloud platform no poly.

Data service is a poly platform service content, data base of geological cloud platform, which can integrate all kinds of geological data source information. Geological data resources include: geological data, geophysical data, geochemical data, remote sensing data and geological disaster data. These raw data sources constitute the geological cloud platform data base services.

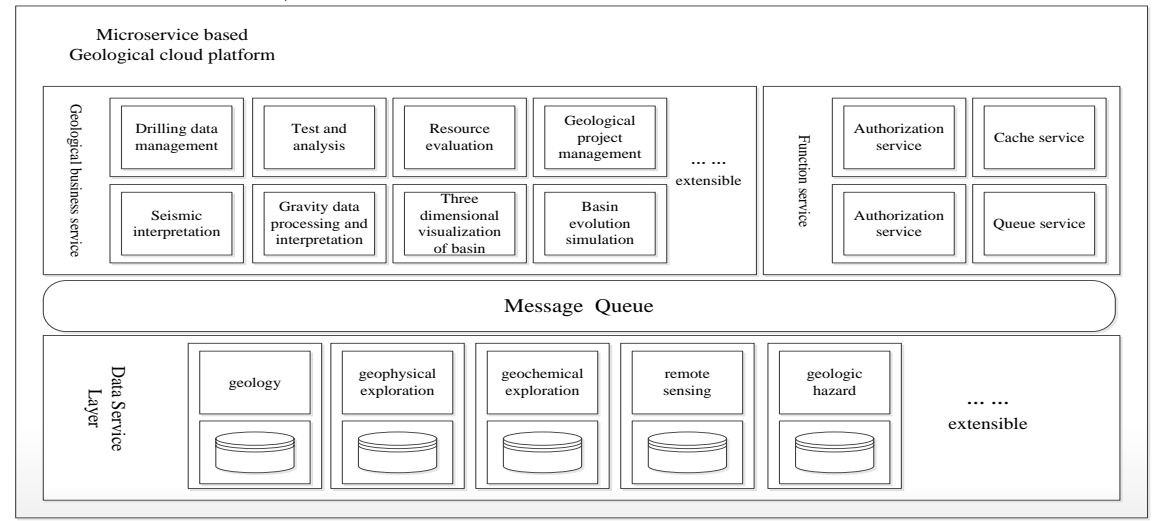

Figure 1. System architecture 


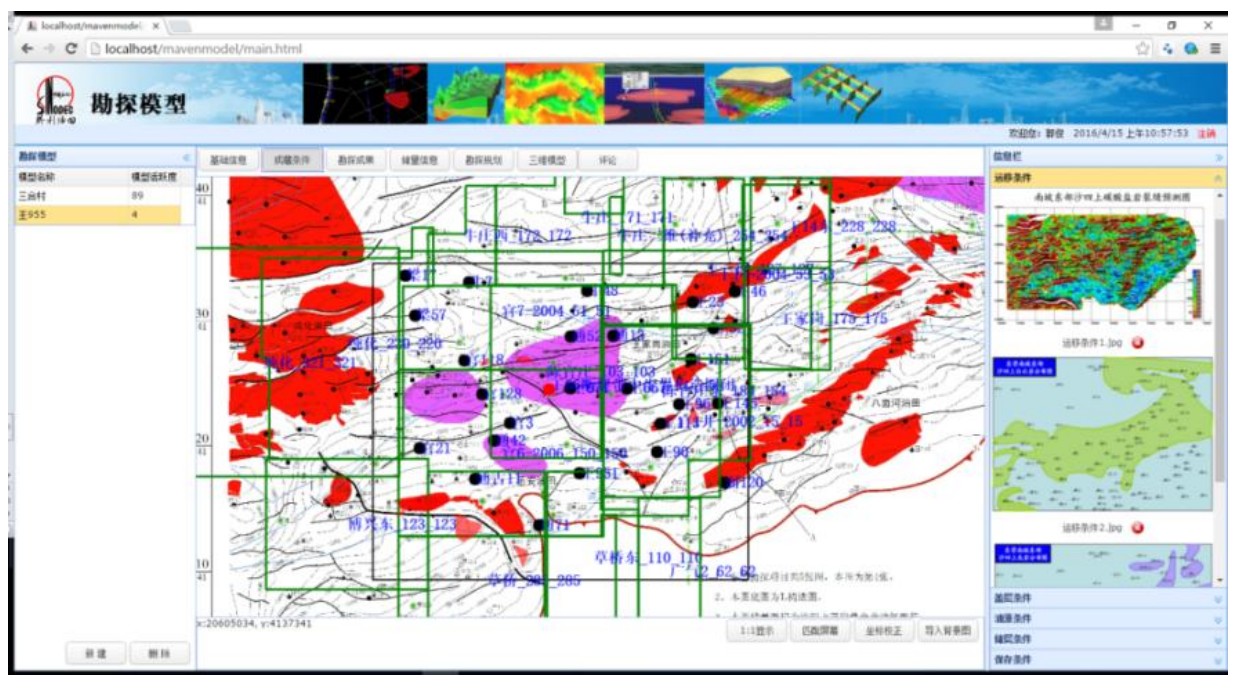

Figure 2. Web application of petroleum geological data web-service

The function of service authorization service, including poly cluster cache service and queue service function, service used mainly as other poly micro service functions, provides caching, authorization and message sending function support.

Business service platform is a poly geological computing services, computing services as geological data services, with each service known as poly data base to provide business users of cloud computing geological services. For example: understanding seismic processing services, processing and interpretation services, gravomagnetic basin visualization services, drilling data management services, testing and analysis of laboratory services and resource evaluation services etc.

\section{B. Service Representation}

Resource representation layer (RRL) as the visualization interface provides a graphical display of data to the end user. It's mainly composed of a cluster of components and devices supported Http 1.1 protocol, such as web browser, smart phone and tablet etc. The software of RRL consists of several web application based on data service, which includes Exploration model web application and QuantyView 3 -demension platform. The former provides visual Web interface based on data entry, combination and management. And implement a geological entity graphical navigation function based on HTML5 canvas. Projection of data entity on 2D geological map, which makes the observation of exploration business object in the exploration area convenient for geological researchers. Moreover, it also helps them to master the panorama and details of the exploration area and serves up the exploration and development preferably. As shown below, the well data, 3D area and reserves traps on the base map projection, in addition, geological maps also in the web interface has been visually display.

Three dimensional geological model based on QuantyView is demonstrated, which provides a 3D visualization environment, and realizes the integration management, processing and application of underground geological, geo spatial and spatial attribute data. It provides application platform for modeling, analysis, design, development and decision of oil and gas basin.

\section{REFERENCE}

[1] Chonglong Wu, Xinqing Wang, Gang Liu. Design principle and application of geological and mineral point source information system [ M] . Wuhan: China University of Geosciences press, 1996.

[2] Jingli Miao,Honggang Qu,Zhe Xu. Study the experimental platform of large data technology GeoBDA[J]. Geographic information world, 2014,21(6):48-52.

[3] BO ETTIGER C. An introduction to Docker for reproducible research[J]. ACM SIGOPS Operating Systems Review, 2015, 49(1) : 71-79.

[4] R.T. Fielding, "Architectural Styles and the Design of Network-based Software Architectures", 2000 http://www.ics.uci.edu/ fielding/pubs/dissertation/top.htm

[5] E. A. Brewer, "Towards Robust Distributed Systems", 2000 http://www.cs.berkeley.edu/ brewer/cs262b-2004/PODC-keynote.pdf

[6] Aitchison J.The Statistical Analysis of Compositional Data(With discussion)[ J].J.R. Statist .Soc.,1982B ,44:139-177.

[7] Perrons,R.K.,J.W.Jensen.Data as an asset:what the oil and gas sector can learn from other industries about "Big Data"[J].Energy Policy,2015.81:p.117-121.

[8] K Holdaway.Harness oil and gas big data with analytics:optimize exploration and production with data driven models[M].2014: John Wiley \& Sons.

[9] Big data solutions \& analytics in upstream oil and gas industry $[\mathrm{C}] .2015$. Oslo,Norway.

[10] Gas,D.A.I.2nd data and information management in oil and gas[C].2015.Abu Dhabi,The United Arab Emirates. 